\title{
Quantum transport through STM-lifted single PTCDA molecules
}

\author{
Florian Pump, ${ }^{1}$ Ruslan Temirov,${ }^{2}$ Olga Neucheva, ${ }^{2}$ Serguei Soubatch, ${ }^{2}$ \\ Stefan Tautz, ${ }^{2}$ Michael Rohlfing, ${ }^{3}$ and Gianaurelio Cuniberti ${ }^{1}$ \\ ${ }^{1}$ Institute for Materials Science and Max Bergmann Center of Biomaterials, \\ Dresden University of Technology, D-01062 Dresden, Germany \\ ${ }^{2}$ Institut für Bio-und Nanosysteme 3, JARA, Forschungszentrum Jülich, D-52425 Jülich, Germany \\ ${ }^{3}$ Department of Physics, University of Osnabrück, D-49069 Osnabrück, Germany
}

(Dated: October 26, 2018)

\begin{abstract}
Using a scanning tunneling microscope we have measured the quantum conductance through a PTCDA molecule for different configurations of the tip-molecule-surface junction. A peculiar conductance resonance arises at the Fermi level for certain tip to surface distances. We have relaxed the molecular junction coordinates and calculated transport by means of the Landauer/Keldysh approach. The zero bias transmission calculated for fixed tip positions in lateral dimensions but different tip substrate distances show a clear shift and sharpening of the molecular chemisorption level on increasing the STM-surface distance, in agreement with experiment.
\end{abstract}

PACS numbers: 68.37.Ef, 81.07.Pr, 73.63.-b, 68.43.Bc, 85.85.+j

\section{INTRODUCTION}

Since the emergence of molecular electronics in the late nineties of the previous century enormous progress has been achieved in both experimental and theoretical understanding of the underlying microscopic mechanisms governing the charge (and also recently spin) migration at the molecular scale [1]. In this sense, the realization of electronic functions at nanometer scales, as pioneered by the visionary work of Aviram and Ratner in 1973 [2], is slowly turning from a dream into a reality. However, there are still formidable challenges to be overcome.

The current state of affairs can be characterized by the following observations: in nanometer-sized junctions the connection between the molecule and the electrode greatly affects the current-voltage characteristics [3], but there are currently no robust methods to image and determine the precise adsorption site and conformation of the molecule on this length scale [4]. We are thus facing a dilemma: on the one hand, we know from theory that contacts are extremely important [5, 6, 7], on the other hand there is - within conventional approaches no way to obtain atomic-scale information on these contacts. The statistical analysis of large data sets (thousands of experiments) has proven to be one way out of the dilemma. However, this procedure is evidently very laborious. For this reason, we wish to explore an alternative approach in the present paper, namely to chemically contact single molecules in a reproducible way, such that reproducible transport spectra can be obtained even in small data sets. This approach is currently being explored; see e.g. refs. 8, 9]. It allows for transport experiments in structurally well-controlled junctions and thus offers an excellent interface to transport calculations. Contacts to single atoms have also been studied [10, 11, 12, 13].

Experimentally, there are three main techniques which form the pillars of the current state-of-the-art: mechanically controllable break junctions (MCBJ) 14, 15, 16,
17, 18, 19, 20, 21, 22, 23], electromigration techniques (EM) 24], and scanning tunnelling microscopy (STM) 8, 25, 26, 27, 28, 29, 30, 31, 32, 33, 34, 35, 36, 37, 38, 39, 40]. Of course, each of these techniques has its specific advantages and drawbacks. For instance, due to their rigid configuration, MCBJs are very stable from a mechanical point of view; consequently, experiments with variable temperatures can be performed. However, MCBJs have no lateral scanning ability and it is very difficult to get information on the detailed microscopic structure of the contacts (see above). Electromigration techniques allow for building three-terminal junctions in a straightforward way, but again the interface to the molecules is not very well defined.

In contrast to the above techniques, STM-based transport experiments in the tip-molecule-surface geometry by virtue of the STM imaging capability - allow for experimental access to the atomic junction structure. However, STM junctions are mechanically less stable and, if operated in the tunnelling regime, they yield intrinsically asymmetric molecular junctions. Moreover, in an STM

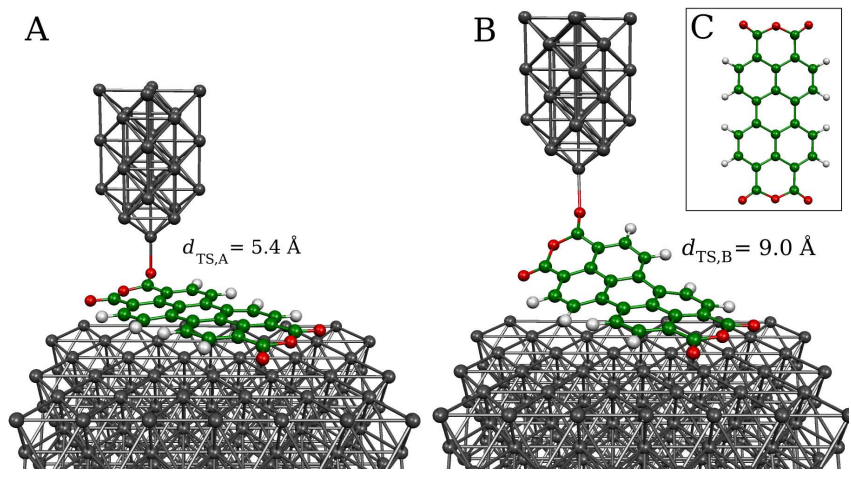

FIG. 1: Relaxed geometries for two different tip substrate distances $d_{\mathrm{TS}}(\mathrm{A}$ and $\mathrm{B}$ ); top view of a PTCDA molecule (C). 
junction electrical gating is often not available, because it is very difficult to realize a third electrode in the vicinity of tip and sample. Finally, temperature dependent transport experiments are more difficult because of thermal drifts of the STM tip with respect to the sample surface. These problems notwithstanding, its is believed that STM-based transport experiments are a worthwhile alternative to MCBJ and EM experiments, because they offer unique advantages, most importantly the structural control. As such, STM-based transport experiments are complementary to the other two techniques. Indeed, the STM tip cannot only address individual molecules, but also specific sub-molecular groups or even individual atoms 9, 12, 41, 42, 43, 44, 45]. Moreover, it must be noted here that some of the disadvantages of the STM approach can in fact be overcome by using the STM in a slightly unconventional fashion: firstly, the contact asymmetry can be surmounted by contacting the molecule with the tip; this contact may either lead to the formation of a strong covalent chemical bond which survives tip retraction ("chemical contact") [8] or be purely "mechanical" in the sense that on tip retraction the contact between tip and molecule is opened again [9]. Secondly, in the case of chemical contacts the tip can also be used for mechanical (in contrast to capacitive) gating by changing the effective coupling strengths between molecule and leads through variations of the vertical tip position [8]. This might potentially allow one to scan different physical regimes ranging from weak coupling (Coulomb blockade), through intermediate coupling (Kondo physics) up to strong coupling (coherent transport). By its capability of manipulation, STM thus allows for tuning of the transport characteristics of individual molecules.

As a way out of the dilemma concerning reproducible molecular electronics experiments mentioned above, we use here a two-step approach based on commensurate, highly ordered molecular layers on metal surfaces [8, 46]. In step 1, the powerful armoury of surface science is employed to characterize the structural and electronic properties of the metal-molecule contact. Once step 1 has been completed, the tip of a low-temperature STM is used to establish a covalent contact with an individual molecule of the highly ordered monolayer (step 2). Because of the excellent imaging properties of the STM, it is in fact possible to select the part of the molecule which is contacted with very high accuracy. In this way a structurally well-defined molecular wire is realized which can be characterized with respect to its transport properties. In favorable cases, it may even be possible to apply a mechanical gating of the wire by tip movements [8].

This paper is organized as follows: Sects. I presents the experimental strategy and the most important results of a proof-of-principle experiment; Sect. III[IV]illustrate how the interface from these experiments to $a b$ initio theory can be devised.

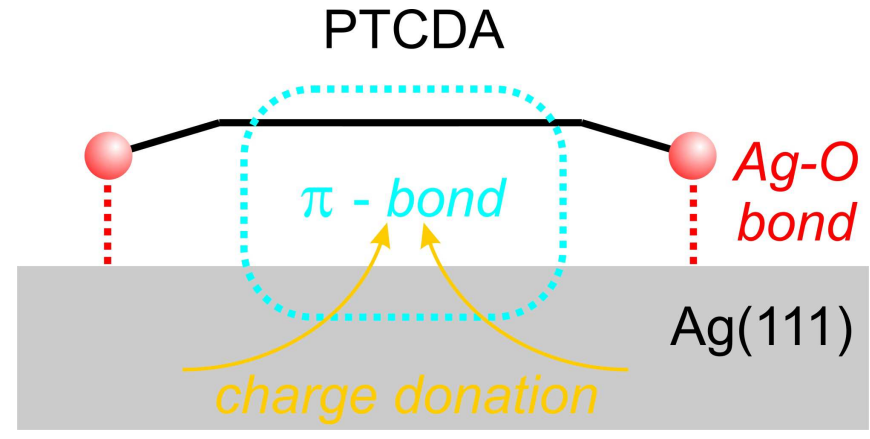

FIG. 2: Schematic representation of the bonding interaction of PTCDA with $\operatorname{Ag}(111)$ [48, 49].

\section{STM EXPERIMENTS}

We have contacted a PTCDA (4,9,10-perylenetetracarboxylic-dianhydride) molecule with an STM tip, peeling it off the $\operatorname{Ag}(111)$ surface and measuring its transport properties in the process. PTCDA $/ \mathrm{Ag}(111)$ is a prototypical model interface, for which the following issues have been addressed by previous work [47]: (1) The precise geometric and electronic structure of the adsorbed monolayer, including site-specific effects and influence of the intermolecular interactions, have been determined (cf. the bonding model in Fig. 2). (2) The mechanisms of the chemical bonding of PTCDA to $\mathrm{Ag}(111)$, including orbital hybridization, charge transfer, local versus extended bonds, and internal molecular distortion have been analyzed. (3) The image formation in the STM has been simulated from first principles. (4) Various density functionals, including GGA and LDA, have been evaluated for the PTCDA/Ag(111) adsorption system. In particular, it must be noted here that according to the bonding model of Fig. 2 the carboxylic oxygen atoms are involved in local Ag-O bonds. By their bond length they effectively define the vertical distance of the perylene core above the metal surface [50]. This in turn controls the degree of metal-molecule hybridization and charge transfer, because both proceed via the extended $\pi$-electron system of the perylene backbone. At the same time, it has been shown that the Ag-O bonds have only a secondary influence on the electronic properties of the adsorbed molecule [8]. To first order, we can thus conclude that the Ag-O bonds act as (by themselves electronically inactive) mechanical clamps which control the electron interaction via the -as we will see below mechanically weaker - perylene-metal bond.

PTCDA/Ag(111) is a system suited well to test the two-step approach outlined at the end of the introduction, because of various reasions. Firstly, the bond of PTCDA to the $\operatorname{Ag}(111)$ substrate is very well characterized, i.e. a lot of experiments with relevance to step 1 have already been performed. Secondly, because of the coexistence of an electronically active but mechanically weak extended bond and an electronically inactive but mechani- 


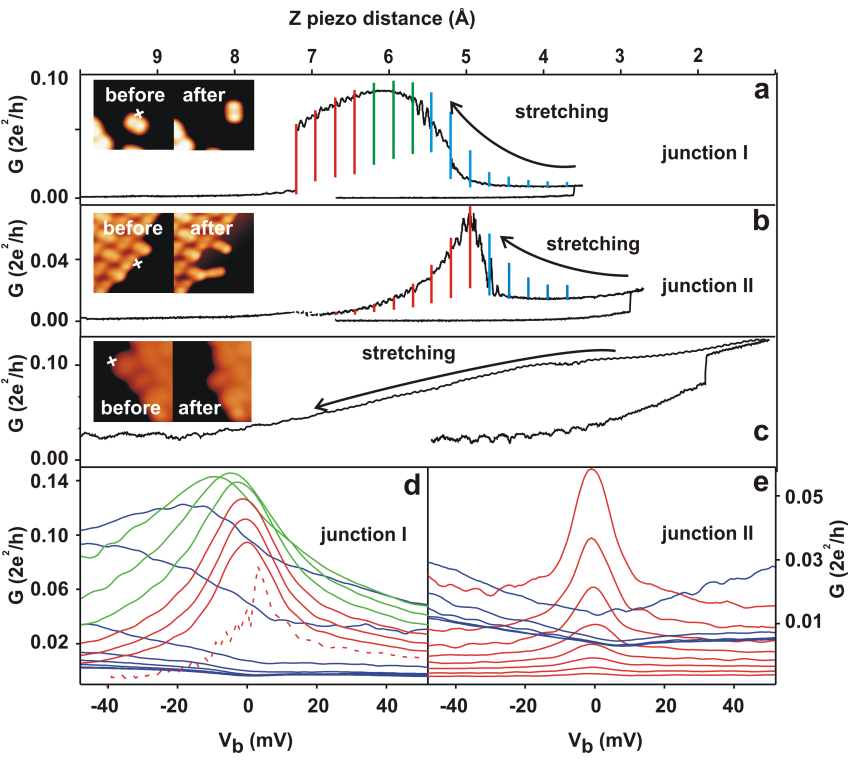

FIG. 3: Approach and retraction spectra of the conductance of the stretched tip-PTCDA-Ag(111) (a, b) or tip-PTCDA$\mathrm{Au}(111)(\mathrm{c})$ transport junctions. Bias voltage $V_{b}=2 \mathrm{mV}$ for (a, b) and $4 \mathrm{mV}$ for (c). Images in (a-c) show the investigated molecule before and after the experiment, the cross indicates the point of approach. The zero of the $z$ scale in (a-c) was established according to the procedure described in Ref. [8]. Vertical bars in $(\mathrm{a}, \mathrm{b})$ : range of differential conductance values from spectra in $(\mathrm{d}, \mathrm{e}))$. (d) Series of $G=d I / d V$ vs. $\left.V_{\mathrm{b}}\right)$ spectra (smoothed) measured at $z$ positions indicated in (a) by vertical bars $\left(V_{\bmod }=4 \mathrm{mV}, \nu_{\bmod }=723 \mathrm{~Hz}\right)$. (e) Series of $G=d I / d V$ vs. $V_{\mathrm{b}}$ spectra (smoothed) measured at $z$ positions indicated in (b) by vertical bars $\left(V_{\bmod }=4 \mathrm{mV}\right.$, $\left.\nu_{\text {mod }}=723 \mathrm{~Hz}\right)$. Colours in $(\mathrm{d}, \mathrm{e})$ code the regimes in $(\mathrm{a}, \mathrm{b})$.

cally strong local bond (see Fig. 21), the molecule offers the possibility for docking the STM tip to the mechanically active terminal of the molecule and gradually cleaving the mechanically weaker, electronic bond to the substrate by tip retraction from the surface, thereby tuning the electronic coupling strength of the molecule to the substrate. We shall see that in effect this indeed amounts to a mechanical gating of transport through the molecular wire. In this simple way a single molecule transport experiment which combines a very high degree of structural control at the contacts with electronic tunability of one of the contacts is devised.

First it must be established that it is indeed possible to contact the PTCDA molecule at one of the carboxylic oxygens. This can be concluded from the $z$-approach spectra, in which the conductance is measured as a function of tip distance from the molecule [8]. If the tip is approached above one of the carboxylic oxygen atoms, a sudden increase of conductance by more than one order of magnitude is observed at a tip distance of approximately $2 \AA$ above the oxygen atom. This step in the conductance is beyond the $z$-resolution of the STM. Since it is only observed above the carboxylic oxygen atoms, we can conclude that at the conductance step the oxygen atom flips up into contact with the tip, to a new potential minimum. Further analysis suggests that the tip-oxygen interaction can only be a covalent bond [8]. For example, the reaction of the tip is independent of the bias direction, which excludes an electrostatic effect. Also, the tip-oxygen contact is strong enough to remove the molecules from the surface. Finally, after jumping into contact, the conductance reaches values which are typical of covalent bonds (in the range of $0.1 G_{0}$ ) 51 With these conductance values, currents through a single molecule can become as large as a few micro-amperes before the molecule is destroyed.

Once the tip-oxygen contact has been formed, it is possible to retract the tip. We have mentioned already that in this way it is possible to remove the molecule from the surface completely. Analyzing the retraction more closely, we find that the conductance remains larger than the corresponding tunnelling values for retraction distances up to approximately $12 \AA[8]$. Hence we can conclude that in this range of tip positions the molecule is still present in the junction. Since the length of the molecule is $12 \AA$, this indicates that the molecule is really lifted from a parallel to a vertical orientation by the tip, i.e. the junction can indeed be stretched out into a vertical wire. On tip retraction, two clearly distinguishable regimes are observed: During the first $4 \AA$, the retraction spectrum is smooth, but in the range from $4 \AA$ to $12 \AA$ it becomes rather jerky. It is clear that if the molecule is lifted up into the vertical, its lower end must at some point slide over the surface, and this is not expected to be a smooth but rather a discontinuous movement.

The possibility to achieve a vertical orientation of the molecule indicates that we are indeed able to modify the contact to the substrate in wide bounds. Most notably, for PTCDA on $\operatorname{Ag}(111)$ we invariably observe that the conductance goes through a maximum within the first $4 \AA$ of tip retraction. To understand the origin of this maximum, we have performed experiments in which tip retraction has been halted at regular intervals to measure full differential conductance spectra in the range from $-50 \mathrm{mV}$ to $50 \mathrm{mV}$. An example of such a series of spectra is shown in Fig. $3 \mathrm{a}$, b, d, e. As the tip is retracted, we observe in the spectra the approach and sharpening of density of states from the left toward the Fermi level. (We note here that for not too strongly coupled wires, the energy-resolved transmission matrix and -accordingly the differential conductance are proportional to the electronic density of states in the wire). This density of states must be assigned to the former LUMO of free PTCDA, which serves as the bonding orbital responsible for the electronic interaction (cf. the bonding sketch in Fig. 2). Its behavior on junction stretching can be explained by reversed chemisorption, i.e. dehybridization of the LUMO from metal states (which leads to sharpening) and an upward shift which follows the one-electron image potential of the occupied orbital (negative charge!) above the metal surface [8]. Incidentally, this interpretation is confirmed by analogous experiments with PTCDA 
on $\mathrm{Au}(111)$, which instead of the conductance maximum always show a monotonous decrease of junction conductance on tip retraction, as shown in Fig. 3. Since the interaction of PTCDA with $\mathrm{Au}(111)$ is merely physisorptive [52, 53], little or no charge transfer between molecule and the $\mathrm{Au}(111)$ substrate occurs, hence the LUMO of PTCDA is never pulled below the Fermi level. Lifting the molecule from the $\mathrm{Au}(111)$ surface thus can only result in a further shift of the LUMO away from the Fermi level (due to decreased polarization screening). The absence of the maximum in the conductance curve for $\mathrm{Au}(111)$ thus indicates that on $\mathrm{Ag}(111)$ it must be linked to the electronic interaction of the molecule with the substrate, as described above.

At the end of the tip retraction experiment, the density of states corresponding to the LUMO becomes pinned at the Fermi level [8]. This cannot be explained in the framework of the reversed chemisorption model, for which one would naively expect the level to be pulled through the Fermi level, following the one-electron image potential further. At least it is difficult to rationalize in a one-particle model why the level should be pinned exactly at the Fermi level, which corresponds to exactly one electron in the orbital. However, if manybody physics is taken into account, there is a mechanism that would naturally explain the observed pinning. This is the Kondo effect. In Ref. [8] we have analyzed the possibility to explain the pinning by the Kondo effect. To this end, an exact solution of the Anderson Hamiltonian [54], using the numerical renormalization group (NRG) [55, 56, 57], has been used. Concerning the choice of the Anderson Hamiltonian as a model for the present system, we recall that in the formulation by Newns [48] this Hamiltonian is widely used as a model for molecular chemisorption on metal surfaces. Indeed it is known from our earlier work that for PTCDA/Ag(111)-chemisorption the LUMO of PTCDA behaves essentially as a local level in the Newns-Anderson model [46, 47, 58]. Accordingly, the Anderson Hamiltonian is an appropriate model for PTCDA/Ag(111), if the role of many-particle correlations is to be analyzed.

The NRG calculation shows that pinning of the bonding orbital at the Fermi level may indeed be observed even outside the fully developed Kondo regime, i.e. at the border to the mixed valence regime [8]. This is an important finding, because our data suggest that on tip retraction the tip/PTCDA/Ag(111) wire remains on the borderline between the Kondo and mixed valence regimes. This can be concluded from the fact that we do not observe a very strong sharpening of the zero bias feature with respect to the single particle peak. (The observed sharpening amounts to a factor of approximately 2 only.) As a consequence, we also do not resolve the two single particle peaks at $\epsilon_{0}$ (singly occupied LUMO) and $\epsilon_{0}+U$ (doubly occupied LUMO) separately, but rather a composite peak which consists of the moderately sharpened many body peak in the center and the two single particle peaks in the left and right tails. In this context it is the most significant finding of the NRG solution that a pinning of the composite resonance to the Fermi level is indeed observed in the borderline regime between mixed valence and Kondo physics - in a parameter range that is suggested by our experiments. The experiment in Fig. 3] shows that before entering the pinning regime $\epsilon_{0}$ roughly amounts to $40 \mathrm{meV}$. Note that the order of magnitude of the intra-orbital Coulomb repulsion which we have employed (100 meV) has been corroborated by a many-body calculation using the GW formalism, which yields $U=0.25 \mathrm{eV}$ for PTCDA on the silver substrate. This value includes a large contribution of the screening by the metal surface: for the free molecule a $U$ of $3.5 \mathrm{eV}$ has been calculated.

In conclusion, the Kondo scenario in our experiments is supported by strong circumstantial evidence. It must be stressed, however, that an experimental test of great significance cannot be performed, since we cannot stabilize the stretched junction at elevated temperatures for times long enough to measure the conductance peak at zero bias. This means that the expected decay of the Kondo peak at temperatures around the Kondo temperature (approximately $120 \mathrm{~K}$ in our case) cannot be verified 8]. However, we note here that in an experiment with $\mathrm{C}_{60}$ in a mechanically controlled break junction [59], in which the $\mathrm{Au}$ contacts transfer charge into the molecule and make the LUMO half-filled, a very similar zero bias conductance peak has been observed on opening the junction, and in that case it could be verified that the peak indeed decays following the dependence predicted for a Kondo peak. Because the transport physics in the experiment by Parks et al. is very similar to the experiment on PTCDA/Ag(111) reported here, it seems permissible to invoke an analogous temperature behavior for our junctions, too. Incidentally, in the experiments by Parks et $a l$. only the pinning regime was observed. The cross-over from the single-particle to the many-particle regime was not reported there.

In spite of the internal consistency of the Kondo scenario outlined above, a final proof is needed. Because of its structurally well-defined nature, the present system offers the unique possibility to provide this proof by a comparison between theory and experiment: Because experimentally the structure of the PTCDA/Ag(111) adsorbate-substrate complex is well known, and because its structural and electronic properties have reliably been calculated by DFT, we can perform transport calculations of the realistic junction. If this is done at various levels of sophistication, the physics of the pinning can be illuminated: if a single-particle calculation does not show pinning, it is very likely that the pinning is a many-body phenomenon. Conversely, if pinning is already observed at the single-particle level, the assumption of Kondo physics may not be necessary, although the mechanism outlined above should still remain valid; its contribution to the observable behavior could then just be dominated by some other mechanism.

In order to realize this program, two types of calcula- 
tions are necessary: First, starting from the experimentally verified structure of the relaxed junction (no tip retraction), the structural and electronic evolution of the junction on tip retraction must be calculated. Secondly, on the basis of the equilibrium structures of the stretched junction, transport calculations may be performed. In the next section we shall turn to a DFT simulation of the process of lifting the molecule off the surface with the retracting STM tip. The results of this simulation will fully corroborate the qualitative picture with respect to contact formation and peel-off deduced from the experiments.

\section{JUNCTION ATOMISTIC CONFIGURATION}

The fundamental questions to be answered by the DFT simulation of the junction stretching process are the following. Firstly: can we verify the flipping up of the carboxylic oxygen atom toward the tip and the formation of a chemical bond between the two? Secondly: what happens to the molecule structurally during tip retraction?

The geometrical configuration of the present system, consisting of a metal substrate, molecule, and STM tip, can successfully be described using density-functional theory (DFT). DFT, as realized by the SIESTA package [60, 61, 62], has already been used by some of the present authors to investigate the adsorption of a monolayer of PTCDA on the $\operatorname{Ag}(111)$ surface [40, 49, 63]. It has turned out that PTCDA adsorbs on bridge sites above the substrate, at an equilibrium height of about $2.8 \AA$, characterized by a nearly flat geometry with a slight molecular distortion. All these features are confirmed by the corresponding experimental data [40, 63], including the partial occupation of the molecular LUMO state by electron charge transfer from the substrate. One should note that DFT as such does not appear to be fully appropriate for the adsorption of PTCDA on $\mathrm{Ag}(111)$ which apart from the chemical interaction has a sizable contribution from physisorption, because long-range van der Waals correlation effects are absent from in DFT [49]. Nonetheless, the theoretical results obtained in Refs. [40, 49, 63] are in close agreement with the measured data, thus justifying the use of DFT for the questions to be studied here. This holds in particular for the local-density approximation (LDA) to the exchange-correlation functional, which yields much better results than the generalized gradient approximation (GGA), which fails to bind the molecule to the surface.

Based on the good results obtained previously for PTCDA on $\mathrm{Ag}(111)$ [49], we here proceed to include an STM tip into the DFT calculation. Since we are not only interested in STM images (which can be described successfully in terms of a Tersoff-Hamann approach, i.e. without explicit inclusion of the tip [49]), we focus on the mechanical interaction at close distances, i.e. we allow for the relaxation of the adsorbed molecules under the influence of forces exerted by the tip and vice versa. To this end, the tip is included as a pyramidal structure of ten tungsten atoms. In close relation to the experimental setup described in Ref. [8], we place the tip above the corner of the molecule, with the tip apex atom exactly above one of the carboxylic oxygen atoms. A chemical bond between the carboxylic oxygen atom and the apex tungsten atom forms, with a bond length of $2.1 \AA$. The calculations thus fully confirm the conclusion drawn previously from our experimental data. Thereafter, the STM tip is lifted up in steps of $0.2 \AA$. After each movement of the tip, the molecule is allowed to relax and follow the tip. One should note that due to the large complexity of the molecule it requires up to 100 geometric relaxation steps until the molecule has adjusted to the retreated tip. Fig. 1 shows two typical relaxed structures of this procedure. In configuration A, the molecule is still close to its free adsorption structure. The carboxylic oxygen atom at the left-hand side of the molecule, however, has formed the chemical bond to the tungsten tip, which has a position of $5.4 \AA$ above the $\mathrm{Ag}$ surface, corresponding to the initial phase of tip retraction. Upon retreating the tip further from the surface, the carboxylic oxygen atom remains chemically bonded to the tip and follows it, causing the molecule to bend and peel off the surface. Fig. 1 $\mathrm{B}$ displays the configuration after retreating the tip by $3.6 \AA$.

The simulation reveals that the junction structure is controlled by three competing energies and interactions. (i) The bond between the carboxylic oxygen and the tip apex atom appears to be quite stiff and to be of a covalent nature. (ii) The molecule is still bonded to the substrate by a delocalized interaction mechanism (cf. Fig. 2). This mechanism is driven by the electron charge transfer from the substrate into the molecular LUMO state, which is delocalized over the entire perylene core of the molecule 49]. This causes attraction between every part of the molecule and the substrate. (iii) The perylene core is relatively soft and can easily be deformed. The sum of all three interaction mechanisms finally yields the curved structure of the molecule in Fig. 1, with its right-hand part still arranged flat on the substrate and the left-hand part lifted off. Apparently, the attraction of the righthand part of the molecule to the surface is still stronger than the energy cost of bending the molecule. Again this is in good agreement with the behavior deduced from experiments. At present, the calculations have not yet reached the regime in which the molecule slides across the surface.

One important feature during the peeling process concerns the occupation of the LUMO state. This can be monitored by projecting the gas-phase LUMO wave function onto the states of the substrate-adsorbate system. Results for three configurations (at tip-surface distances of $5.4 \AA, 6.8 \AA$, and $9.0 \AA$ ) are displayed in Fig. 4. These are the configurations for which transport calculations will be discussed further below. It is clearly visible that, in accordance with the mechanism as described above, the LUMO is shifted to higher energy during the peeling 


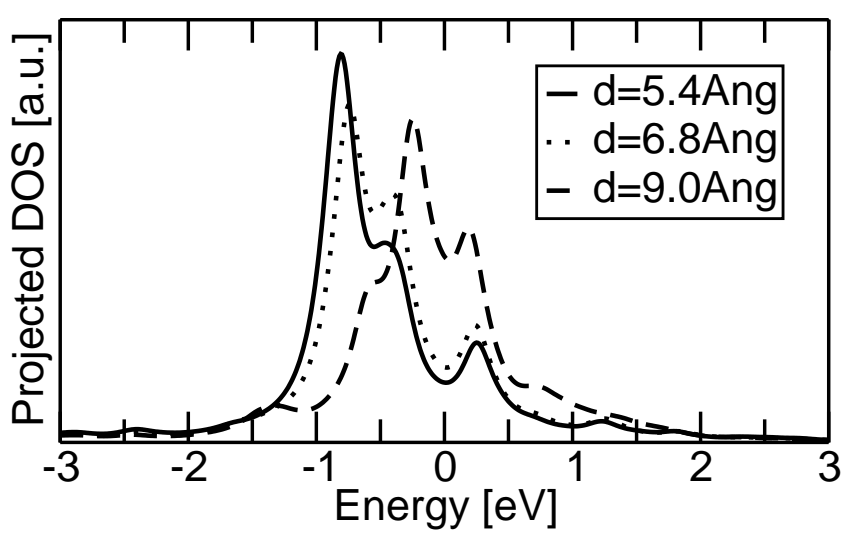

FIG. 4: Density of states ofthe LUMO state, projected onto the electronic states of the substrate-adsorbate complex, for configurations obtained at tip-surface distances of $5.4 \AA, 6.8$ $\AA$, and $9.0 \AA$.

process, and at $9.0 \AA$ tip height it has already a substantial density of states above the Fermi level. This is accompanied by successive de-population and spectral sharpening. The completely removed molecule would have a sharp LUMO above the Fermi level of the substrate.

The geometries as determined by the DFT simulation of the lift-off are the basis for the transport calculations described in the next section.

\section{DFT-BASED COHERENT TRANSPORT CALCULATIONS}

As a further step towards a deeper understanding of the transport properties described in Sec. [II we perform transport simulations through a junction composed of substrate, molecule and STM tip. Even though modelbased approaches often give valuable insight into underlying transport mechanisms [64, 65, 66, 67], a realistic, material-specific description can only be achieved by an atomistic study. Given the size of the system, an approximate density functional parametrized tight-binding method, DFTB, is used to account for the electronic structure of the hybrid system. The input geometries for these calculations are the fully relaxed ones obtained using the DFT package SIESTA as described above in Sec. III. to obtain optimal reliability of the structure.

As a first step in the transport simulations, we show in the present study as a characterizing feature the transmission function $T(E)$ at zero bias voltage for different distances between the substrate and the STM tip. In this way one can follow the evolution of the molecular levels (peaks in the transmission spectra) and the electronic coupling to the contacts (metallic surface and STM tip, respectively) which is reflected by the width of these peaks. To do so, we use as computational method the quantum transport implementation gDFTB [68, 69], which is a combination of the non-equilibrium Green function technique to describe the transport process and a DFT-based two-center charge self-consistent tightbinding method, DFTB [70], to account for the electronic structure of the extended molecular system (see Fig. 1). During the transport calculation, starting from the system Hamiltonian, a charge self-consistency loop over the non-equilibrium Green function, the density matrix and the real space charge density is performed under the nonequilibrium conditions imposed by the applied bias voltage and the attached contacts. The resulting electric field is calculated via solving the Poisson equation at any loop cycle. The electric contacts are modeled as ideal semiinfinite wires, which are projected by decimation techniques onto an effective surface Green function. As the transport property considered here, the coherent transmission function is given by

$$
T(E)=\operatorname{Tr}\left[\Gamma_{1} G^{\mathrm{r}} \Gamma_{2} G^{\mathrm{a}}\right],
$$

where $\Gamma_{1}$ and $\Gamma_{2}$ describe the coupling between the electrodes and the extended molecule at the contact layers. $G^{\mathrm{r}}$ and $G^{\mathrm{a}}$ are the retarded and advanced Green function of the extended molecule which are calculated as the result of the self-consistency loop.

It has to be stressed that the presented calculations are single-particle ones and cannot capture typical Kondo physics features or charging effects. However, the results give deep insight into the electronic structure and its evolution during the lifting process and provide hints as to whether the observed experimental features can be explained in a single-particle picture or need a more sophisticated explanation.

In the transport calculations, a single PTCDA molecule, one surface layer consisting of 38 atoms and 10 atoms of the top of the STM tip are taken as the extended molecule to be treated fully within DFTB for taking into account charge transfer processes between the surface and the molecule at all stages of the lifting process. Both leads are modeled by semi-infinite silver wires. It has to be noted that finite size effects may lead to a quantitative difference compared to studies of the system taking into account periodic boundary conditions which are currently carried out. The parameter sets used in this study were the ones available from the DFTB project [70, 71, 72, 73]. Fig. [5 shows the zero bias transmission function $T$ as a function of the incoming particle energy $E$ for different tip-substrate distances. It can clearly be seen that the spectrum, especially the peak closest to the Fermi energy, moves in a reversed chemisorption process in a positive energy direction as observed in experiment. For the largest distance investigated in the present study, i.e. $9.0 \AA$, the peak is located closely below the Fermi energy. The further evolution of the position of this peak with larger distances (the molecule lifted further from the surface) is of great interest as it could show whether the level crosses the Fermi energy or if it remains pinned there. The relaxation of the system for this parameter regime is currently under investigation. Furthermore it is evident from Fig. 5 that 


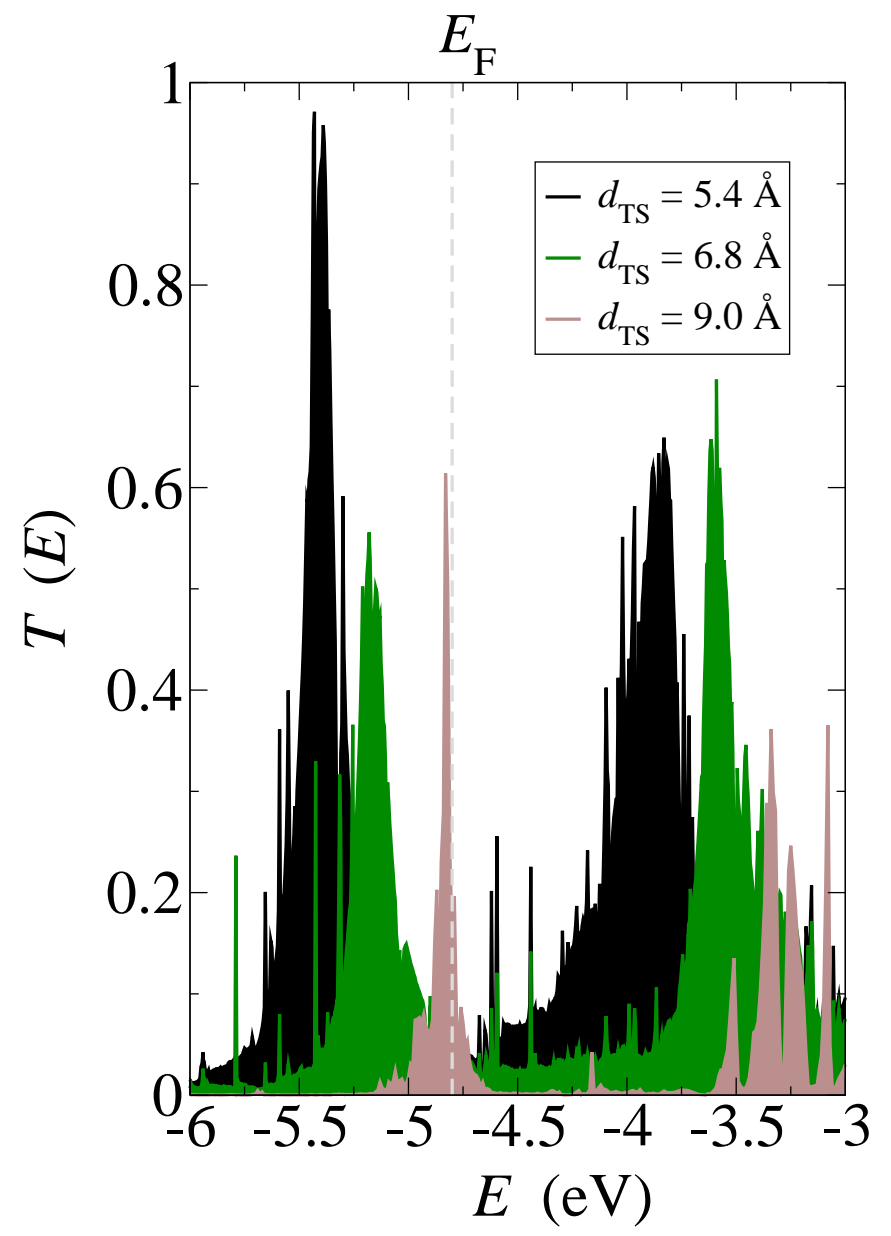

FIG. 5: Transmission at zero bias as a function of energy for different tip substrate distances $d_{\mathrm{TS}}$.

the width of the peaks in the spectra is decreased for larger distances, as, due to a reduction of the overlap of the molecular $\pi$-system with the metal electronic system, the electronic coupling strength and hence the injection rate between the molecule and the metallic substrate is reduced (Breit-Wigner physics).

\section{CONCLUSIONS AND OUTLOOK}

Using quantum transport methods, we have shown the motion of the energy levels in positive direction, espe- cially of the frontier orbitals when lifting the molecule from the substrate.

In continuation of the present work, it is necessary to explore stable system setups with larger tip-substrate distances to follow the whole trace of the molecule during the lifting process as performed in the experiments, where also lateral movement of the molecules on the surface is observed. Furthermore, an investigation of the different behavior and properties comparing isolated molecules on a surface to a monolayer of PTCDA is presently performed. To finally clarify the origin of the experimentally observed features, it is necessary to find clear relations between the important magnitudes governing transport and the experimental input parameter, the tip substrate distance. In detail, these are the charging energy $U$, the coupling strengths between molecule and substrate, described by $\Gamma$, and the level energy $\epsilon$. Depending on their functional dependence during the lifting process, it will be possible to decide whether a single particle picture is sufficient to describe the observations or if a Kondo picture needs to be applied.

Quite apart form the question whether the Kondo scenario is applicable for our model system, our proof-ofprinciple experiment and the simulation program, carried out here for PTCDA, provide a benchmark for the comparison of a single-molecule transport experiment and $a b$-initio transport simulations, because hardly ever is structural information on the transport junction available that is so detailed.

\section{ACKNOWLEDGEMENTS}

This work was partially funded by the Volkswagen Foundation (contract I/78 340), the German Research Foundation (DFG) Priority Program 1243 "Quantum transport at the molecular scale". The Center for Information Services and High Performance Computing (ZIH) at the Dresden University of Technology provided computation time. We are indebted to Aldo Di Carlo and Alessandro Pecchia for the gDFTB code and to Alessio Gagliardi, Rafael Gutiérrez and Bo Song for fruitful discussions.
[1] G. Cuniberti, G. Fagas and K. Richter, Introducing Molecular Electronics: A brief overview, vol. 680 of Lecture Notes in Physics (Springer, 2005).

[2] A. Aviram and M. A. Ratner, Chem. Phy. Lett. 29(2), 277 (1974).

[3] A. Nitzan and M. A. Ratner, Science 300, 1384 (2003).

[4] C. Joachim and M. A. Ratner, Proc. Natl. Acad. Sci.
USA 102, 8800 (2005).

[5] M. del Valle, R. Gutiérrez, C. Tejedor, and G. Cuniberti, Nature Nanotech. 2, 176 (2007).

[6] S.-H. Ke, H. U. Baranger, and W. Yang, J. Chem. Phys. 123, 114701 (2005).

[7] C.-C. Kaun and T. Seideman, Phys. Rev. B 77, 033414 (2008). 
[8] R. Temirov, A. C. Lassise, F. Anders, and F. S. Tautz, Nanotechnology 19, 065401 (2008).

[9] N. Néel, J. Kröger, L. Limot, T. Frederiksen, M. Brandbyge, and R. Berndt, Phys. Rev. Lett. 98, 065502 (2007).

[10] N. Néel, J. Kröger, L. Limot, J. Palotas, W. Hofer, and R. Berndt, Phys. Rev. Lett. 98, 016801 (2007).

[11] J. Kröger, H. Jensen, and R. Berndt, New J. Phys. 9, 153 (2007).

[12] L. Limot, J. Kröger, R. Berndt, A. Garcia-Lekue, and W. A. Hofer, Phys. Rev. Lett. 94, 126102 (2005).

[13] A. Yazdani, D. M. Eigler, and N. D. Lang, Science 272, 1921 (1996).

[14] M. Dreher, F. Pauly, J. Heurich, J. C. Cuevas, E. Scheer, and P. Nielaba, Phys. Rev. B 72, 075435 (2005).

[15] E. Scheer, P. Joyez, D. Esteve, C. Urbina, and M. H. Devoret, Phys. Rev. Lett. 78, 3535 (1997).

[16] E. Scheer, N. Agrait, J. C. Cuevas, A. L. Yeyati, B. Ludoph, A. Martin-Rodero, G. R. Bollinger, J. M. van Ruitenbeek, and C. Urbina, Nature 394, 154 (1998).

[17] M. Elbing, R. Ochs, M. Koentopp, M. Fischer, C. von Hanisch, F. Weigend, F. Evers, H. B. Weber, and M. Mayor, Proc. Natl. Acad. Sci. USA 102(25), 8815 (2005).

[18] J. Reichert, R. Ochs, D. Beckmann, H. B. Weber, M. Mayor, and H. v. Löhneysen, Phys. Rev. Lett. 88, 176804 (2002).

[19] J. Reichert, H. B. Weber, M. Mayor, and H. v. Lohneysen, Appl. Phys. Lett. 82, 4137 (2003).

[20] H. B. Weber, R. Häussler, H. v. Löhneysen, and J. Kroha, Phys. Rev. B 63, 165426 (2001).

[21] F. Q. Xie, L. Nittler, C. Obermair, and T. Schimmel, Phys. Rev. Lett. 93, 128303 (2004).

[22] R. H. M. Smit, Y. Noat, C. Untiedt, N. D. Lang, M. C. van Hemert, and J. M. van Ruitenbeek, Nature 419, 906 (2002).

[23] A. Agrait, A. Levy-Yeyati, and J. M. van Ruitenbeek, Phys. Rep. 377, 81 (2003).

[24] H. B. Heersche, Z. de Groot, J. A. Folk, L. P. Kouwenhoven, H. S. J. van der Zant, A. A. Houck, J. Labaziewicz, and I. L. Chuang, Phys. Rev. Lett. 96, 017205 (2006).

[25] J. Repp, G. Meyer, S. Paavilainen, F. E. Olsson, and M. Persson, Phys. Rev. Lett. 95, 225503 (2005).

[26] B. C. Stipe, M. A. Rezaei, and W. Ho, Phys. Rev. Lett. 81, 1263 (1998).

[27] B. C. Stipe, M. A. Rezaei, and W. Ho, Phys. Rev. Lett. 82, 1724 (1999).

[28] B.-Y. Choi, S.-J. Kahng, S. Kim, H. Kim, H. Won Kim, Y. J. Song, J. Ihm, and Y. Kuk, Phys. Rev. Lett. 96, 156106 (2006).

[29] H. Yanagi, K. Ikuta, H. Mukai, and T. Shibutani, Nano Letters 2, 951 (2002).

[30] K.-F. Braun and S.-W. Hla, Nano Letters 5, 73 (2005).

[31] S.-W. Hla, K.-F. Braun, B. Wassermann, and K.-H. Rieder, Phys. Rev. Lett. 93, 208302 (2004).

[32] V. Iancu, A. Deshpande, and S.-W. Hla, Nano Lett. 6, 820 (2006).

[33] J. Nieminen, S. Lahti, S. Paavilainen, and K. Morgenstern, Phys. Rev. B 66, 165421 (2002) (2002).

[34] V. Simic-Milosevic, M. Mehlhorn, K.-H. Rieder, J. Meyer, and K. Morgenstern, Phys. Rev. Lett. 98, 116102 (2007).

[35] A. J. Mayne, M. Lastapis, G. Baffou, L. Soukiassian, G. Comtet, L. Hellner, and G. Dujardin, Phys. Rev. B 69, 045409 (2004).
[36] G. Dujardin, A. J. Mayne, and F. Rose, Phys. Rev. Lett. 89, 036802 (2002).

[37] M. Martin, M. Lastapis, D. Riedel, G. Dujardin, M. Mamatkulov, L. Stauffer, and P. Sonnet, Phys. Rev. Lett. 97, 216103 (2006).

[38] N. Knorr, M. A. Schneider, L. Diekhöner, P. Wahl, and K. Kern, Phys. Rev. Lett. 88, 096804 (2002).

[39] P. Wahl, L. Diekhöner, G. Wittich, L. Vitali, M. A. Schneider, and K. Kern, Phys. Rev. Lett. 95, 166601 (2005).

[40] A. Kraft, R. Temirov, S. K. M. Henze, S. Soubatch, M. Rohlfing, and F. S. Tautz, Phys. Rev. B 74, 041402(R) (2006).

[41] J. A. Stroscio and R. J. Celotta, Science 306, 242 (2004).

[42] D. M. Eigler, C. P. Lutz, and W. E. Rudge, Nature 352, 600 (1991).

[43] D. M. Eigler and E. K. Schweizer, Nature 344, 524 (1990).

[44] H. J. Lee and W. Ho, Science 286, 1719 (1999).

[45] J. Repp, G. Meyer, S. M. Stojkovic, A. Gourdon, and C. Joachim, Phys. Rev. Lett. 94, (2005).

[46] S. Soubatch, R. Temirov, and F. S. Tautz, Phys. Stat. Sol. A 205, 511 (2008).

[47] F. S. Tautz, Prog. Surf. Sci. 82, 479 (2007).

[48] D. M. Newns, Phys. Rev. 178, 1123 (1969).

[49] M. Rohlfing, R. Temirov, and F. S. Tautz, Phys. Rev. B 76, 115421 (2007).

[50] L. Kilian, A. Hauschild, R. Temirov, S. Soubatch, A. Schöll, A. Bendounan, R. Reinert, F. S. Tautz, M. Sokolowski, and E. Umbach, Phys. Rev. Lett. 100, 136103 (2008).

[51] Z. Li, B. Han, G. Meszaros, I. Pobelov, T. Wandlowski, A. Blaszczyk, and M. Mayor, Faraday Discussions 131, 121 (2006).

[52] S. K. M. Henze, O. Bauer, T. L. Lee, M. Sokolowski, and F. S. Tautz, Surf. Sci. 601, 1566 (2007).

[53] M. Eremtchenko, D. Bauer, J. A. Schäfer, and F. S. Tautz, New J. Phys. 6, 4 (2004).

[54] P. W. Anderson, Phys. Rev. 124, 41 (1961).

[55] H. R. Krishna-Murthy, J. W. Wilkins, and K. G. Wilson, Phys. Rev. B 21, 1003 (1980).

[56] R. Peters, T. Pruschke, and F. B. Anders, Phys. Rev. B 74, 245114 (2006).

[57] A. Weichselbaum and J. v. Delft, Phys. Rev. Lett. 99, 076402 (2007).

[58] A. Kraft, R. Temirov, S. K. M. Henze, S. Soubatch, M. Rohlfing, and F. S. Tautz, Physical Review B 74, 041402 (2006).

[59] J. J. Parks, A. R. Champagne, G. R. Hutchison, S. Flores-Torres, H. D. Abruña, and D. C. Ralph, Phys. Rev. Lett. 99, 026601 (2007).

[60] For details about the SIESTA package please refer to http://www.uam.es/departamentos/ciencias/fismateriac/

[61] P. Ordejon, E. Artacho, and J. M. Soler, Phys. Rev. B 53, R10441 (1996).

[62] J. M. Soler, E. Artacho, J. D. Gale, A. Garcia, J. Junquera, P. Ordejon, and D. Sanchez, J. Phys.-Condens. Matter 14, 2745 (2002).

[63] A. Hauschild, K. Karki, B. C. C. Cowie, M. Rohlfing, F. S. Tautz, and M. Sokolowski, Phys. Rev. Lett. 94, 036106 (2005).

[64] R. Gutiérrez, S. Mandal, and G. Cuniberti, Nano Lett. 5, 1093 (2005).

[65] F. Pump and G. Cuniberti, Surf. Sci. 601, 4109 (2007). 
[66] B. Song, D. A. Ryndyk, and G. Cuniberti, Phys. Rev. B 76, 045408 (2007).

[67] D. A. Ryndyk, P. D'Amico, G. Cuniberti, and K. Richter, Phys. Rev. B 78, 085409 (2008).

[68] A. Pecchia and A. Di Carlo, Rep. Prog. Phys. 67, 1497 (2004).

[69] A. Di Carlo, A. Pecchia, L. Latessa, T. Frauenheim, and G. Seifert, Chapter 5 in [1] .

[70] M. Elstner, D. Porezag, J. Elsner, G. Jungnickel,
M. Haugk, T. Frauenheim, S. Suhai, and G. Seifert, Phys. Rev. B 58, 7620 (1998).

[71] B. Szuecs, Z. Hajnal, T. Frauenheim, C. González, J. Ortega, R. Pérez, and F. Flores, Appl. Surf. Sci. 212, 861 (2003).

[72] B. Szuecs, Z. Hajnal, R. Scholz, S. Sanna, and T. Frauenheim, Appl. Surf. Sci. 234, 173 (2004).

[73] Please refer to http://www.dftb.org 